\title{
Prevalence of UL97 gene mutations in cytomegalovirus reactivation in the colon associated with or without ulcerative colitis
}

\section{Satoshi Tamura}

Hamamatsu University School of Medicine

Satoshi Osawa ( $\sim$ sososawa@hama-med.ac.jp)

Hamamatsu University School of Medicine

\section{Natsuki Ishida}

Hamamatsu University School of Medicine

\section{Takahiro Miyazu}

Hamamatsu University School of Medicine

\section{Shinya Tani}

Hamamatsu University School of Medicine

Mihoko Yamade

Hamamatsu University School of Medicine

Moriya Iwaizumi

Hamamatsu University School of Medicine

Yasushi Hamaya

Hamamatsu University School of Medicine

Isao Kosugi

Hamamatsu University School of Medicine

Takahisa Furuta

Hamamatsu University School of Medicine

Ken Sugimoto

Hamamatsu University School of Medicine

\section{Research Article}

Keywords: cytomegalovirus colitis, ganciclovir resistance, polymorphisms, UL97 gene, ulcerative colitis

Posted Date: April 21st, 2021

DOl: https://doi.org/10.21203/rs.3.rs-92850/v2 
License: (c) (i) This work is licensed under a Creative Commons Attribution 4.0 International License. Read Full License 


\section{Abstract}

Cytomegalovirus (CMV) reactivation in the colon is common in patients with severe ulcerative colitis (UC). Ganciclovir (GCV) resistance conferring CMV UL97 gene mutations have been reported in recent years. However, the prevalence of UL97 gene mutations in GCV-naive CMV infection in the colon remains unknown. We investigated the prevalence of CMV UL97 gene mutations in patients with colonic CMV infection associated with or without UC. Twenty-two GCV-naive patients with colonic CMV infection, 15 with UC and 7 with other diseases, were enrolled. Frozen biopsy samples or formalin-fixed paraffinembedded samples were used for nested polymerase chain reaction (PCR) amplification of the UL97 gene. Sanger DNA sequencing was performed. UL97 mutations were frequently detected in codons T75A (95.5\%), Q126L (86.4\%), and D605E (86.4\%), and less frequently (<10\%) in L228P, D263G, A53S, R137C, A140V, G188S, A674T, and T675A. However, no known GCV resistance mutations were found. There seemed to be no difference between the ratio of mutations in patients with and without UC. In conclusions, we did not detect UL97 gene mutations associated with GCV resistance in GCV-naive patients with or without UC. T75A, Q126L, and D605E mutations may be used as genetic markers for $\mathrm{CMV}$ in East Asian countries.

\section{Background}

Human cytomegalovirus (CMV) infections cause significant morbidity and mortality in immunocompromised hosts, such as patients who have undergone solid organ or bone marrow transplantation, human immunodeficiency virus (HIV)-infected patients, and children with congenital immunodeficiencies ${ }^{1,2}$. In patients with flareups of refractory ulcerative colitis (UC) and sometimes in immunocompromised patients, CMV reactivation in the colon is common and may be associated with poor prognosis ${ }^{3}$. In immunocompetent individuals, CMV usually causes self-limiting mild hepatitis, mononucleosis, or subclinical infection ${ }^{4}$. Antiviral treatment with ganciclovir (GCV) has been highly recommended for CMV reactivation, along with anti-tumor necrosis factor (anti-TNF) monoclonal antibody therapy, without losing time, even though there is some debate recently in flareups of refractory UC $^{5-9}$.

GCV, a 2'-deoxyguanosine nucleoside analog, was used as the first-line drug for the treatment of CMV disease and for prophylaxis in groups at high risk for CMV infection. However, prolonged therapy with GCV can lead to the development of GCV-resistant mutations ${ }^{10,11}$. GCV is selectively phosphorylated by a viral protein kinase homolog, a product of the UL97 gene ${ }^{12,13}$. Approximately $90 \%$ of GCV resistance results from mutations in that gene ${ }^{13}$. Well characterized GCV resistance mutations at UL97 codons 460, 520, and 590-607 impair the phosphorylation of GCV that is necessary for its antiviral activity,

presumably by altering substrate recognition ${ }^{14-16}$. Despite their lack of association with GCV resistance, some additional mutation sites of UL97 have also been reported ${ }^{17,18}$. 
Previous studies have revealed the frequency of drug-resistant CMV in organ transplant patients, bone marrow transplant patients, and HIV-infected patients, and have discussed algorithms for antiviral

therapy ${ }^{11,19}$. However, there are no data regarding the prevalence of UL97 gene mutations in colonic CMV infection associated with or without UC. One of the clinical questions is whether or not the uniform use of GCV is appropriate for initial antiviral therapy. The aim of this study was to investigate the prevalence of GCV resistance-conferring UL97 gene mutations in Japanese patients with colonic CMV infection associated with or without UC, especially in GCV-naive patients.

\section{Methods}

\section{Study design}

This was a single-center, retrospective study conducted in patients with colonic CMV infections with and without UC. This study was conducted in accordance with the Declaration of Helsinki, and the study protocol was reviewed and approved by the Ethics Committee of Hamamatsu University School of Medicine, Japan (EG16-257). Informed consent was omitted, and information of this study was disclosed in the form of an opt-out on our hospital website. Information regarding the conduct of the research, including the objectives, was disclosed, and the research subjects were provided an opportunity to refuse inclusion in the research.

\section{Patients and specimens}

Twenty-two GCV-naive patients who were diagnosed with colonic CMV infection in our hospital between 2012 and 2018 were enrolled in this study. Colonic CMV infection was defined as virus isolation or detection of viral proteins (antigens) or nucleic acid in colonic tissue specimen ${ }^{20}$. Fifteen patients with CMV infection in the colon had UC, and seven patients had other diseases. Frozen biopsy samples or formalin-fixed paraffin-embedded samples were used for nested polymerase chain reaction (PCR) amplification of the UL97 gene.

\section{DNA purification from the samples}

Frozen biopsy samples were used for DNA extraction using the DNeasy Blood and Tissue Kit (Qiagen, Germantown, MD). Formalin-fixed paraffin-embedded samples were purified genomic DNA using the QIAamp DNA FFPE Tissue Kit (Qiagen). The extractions were carried out according to the manufacturer's instructions.

\section{Nested PCR amplification}


All PCR primers were purchased from Merck KGaA (Darmstadt, Germany). Nested PCR was performed with KOD FX neo (TOYOBO CO., LTD, Osaka, Japan). The primer sets used in the nested PCR for this study are shown in Fig. 1 and Table 1. The UL97 fragment was amplified using $1.5 \mathrm{mM} \mathrm{MgCl}$, $0.2 \mathrm{mM}$ dNTP, 1 U Taq DNA polymerase, 10X PCR buffer, $300 \mathrm{nM}$ of each primer, and $50 \mathrm{ng}$ genomic DNA of CMV. For the first round of PCR, the thermal cycler was run at $98^{\circ} \mathrm{C}$ for $10 \mathrm{~s}, 58^{\circ} \mathrm{C}$ for $30 \mathrm{~s}$ and $72^{\circ} \mathrm{C}$ for $30 \mathrm{~s}$ for 25 cycles, then $72^{\circ} \mathrm{C}$ for $7 \mathrm{~min}$. For the second round of PCR, the thermal cycler was run at $98^{\circ} \mathrm{C}$ for $10 \mathrm{~s}, 62^{\circ} \mathrm{C}$ for $30 \mathrm{~s}$, and $72^{\circ} \mathrm{C}$ for $30 \mathrm{~s}$ for 25 cycles, then $72^{\circ} \mathrm{C}$ for $7 \mathrm{~min}$. The QIAquick PCR Purification Kit (Qiagen) was used to purify the PCR products. Reaction products were stored at $-20^{\circ} \mathrm{C}$ until they were analyzed by $2 \%$ agarose gel electrophoresis or purified for DNA sequencing.

\section{Sanger DNA sequencing and data analysis}

Sanger sequence was performed using an Applied Biosystems 3130 and 3500xL Genetic Analyzer (ThemoFisher Scientific, Waltham, MA). Established sequences were compared to the UL97 gene of the wild-type GCV-sensitive Towne strain (GenBank NC_006273.2) as a reference.

\section{Statistical analysis}

Statistical analysis was performed using statistical software (SPSS for Windows, version 16.0; SPSS Inc., Chicago, IL). The Fisher's exact test was used to compare categorical variables between the UC and non-UC groups. The Mann-Whitney Utest and independent Student's $t$-test were used to compare continuous variables of the UC and non-UC groups, as appropriate. The Fisher's exact test was used to compare each mutation frequency in the UL 97 gene between the UC and non-UC groups. A $P$-value of $<0.05$ was considered significant.

\section{Results}

\section{Patient characteristics}

The demographic information for $22 \mathrm{GCV}$-naive patients enrolled in this study is shown in Table 2. The mean age was 59.5 years. Fifteen patients had UC and seven patients had other diseases. In non-UC patients, the background diseases were malignant melanoma (one patient), myelodysplastic syndrome (one patient), sarcoidosis (one patient), ischemic colitis (one patient), autoimmune hepatitis (one patient), and hemodialysis (one patient). A comparison of the patient characteristics between the UC and non-UC groups are shown in Table 3. In the comparison of the two groups, serum albumin was significantly lower in the non-UC group. Among the treatments, there was a significant difference only in the use of 5aminosalicylate (5-ASA) (Table 3). Among the UC patients, steroid therapy was used for five patients (33.3\%), immunosuppressive therapy was used for seven patients (46.7\%), anti-TNF therapy was used for 
five patients (33.3\%), and 5-ASA was used for 10 patients (66.7\%). The clinical UC activity of the enrolled patients assessed by the Rachmilewitz index was $8.75 \pm 5.39$ (Supplementary Table 1).

\section{Diagnosis of CMV infection in the colon}

In our study, CMV infection in the colon was diagnosed on the basis of mucosal biopsies with macroscopic inflammation. Infected cells with intracellular inclusion bodies were detected in 4 of 22 patients by hematoxylin and eosin staining in tissue specimens (Table 3). Histological IHC staining revealed that 19 of 22 patients were CMV positive. All patients were CMV positive by the tissue CMV DNA PCR method.

\section{Prevalence of the UL97 gene mutation}

To identify the mutation of the UL97 gene in GCV-naive patients, Sanger DNA sequencing of the PCR products was performed. Compared with the wild-type GCV-sensitive Towne strain as a reference, we were able to detect several mutations of the UL97 gene. Typical results of identified UL97 genetic mutations are shown in Fig. 2. Overall, in 22 patients with CMV reactivation in the colon, UL97 mutations were frequently detected in codons T75A (95.5\%), Q126L (86.4\%), and D605E (86.4\%), and less frequently in L228P (9.0\%), D263G (9.0\%), A53S (4.5\%), R137C (4.5\%), A140V (4.5\%), G188S (4.5\%), A674T (4.5\%), and T675A (4.5\%). T75A and Q126L have not been reported previously (Table 4). However, no known GCV resistance mutations were found in our series.

\section{Comparison between UC and non-UC patients}

We attempted to examine whether the frequency and type of UL97 mutations in GCV-naive patients were different between UC and non-UC patients. As shown in Table 4, the ratio of the mutations seemed to be similar between the UC group and non-UC group although the number of sample was insufficient.

\section{Discussion}

In the present study, we investigated the prevalence of CMV UL97 gene mutations in Japanese patients with CMV reactivation in the colon for the first time. We revealed that several UL97 mutations were frequently detected, such as in codons Q126L (100\%), T75A (93.3\%), and D605E (80\%). Q126L and T75A have not been reported previously. However, no known GCV resistance mutations were found in our series of GCV-naive patients, and, furthermore, there was no difference between the ratio of mutations in the UC 
and the non-UC patients. The Q126L, T75A, and D605E mutations could be used as genetic markers for CMV in East Asian countries because UL97 mutations were suggested to have regional differences.

Human CMV remains the most common infection in solid organ recipients, HCT recipients, HIV-infected patients, and children with congenital immunodeficiencies. It remains an important pathogen despite advances in the prophylaxis and acute treatment of CMV. The emergence of CMV resistance in a patient reduces the clinical efficacy of antiviral therapy, complicates therapeutic and clinical management decisions, and, in some cases, results in death of the patient. According to recent reports, the incidence of GCV resistance is $5-12 \%$ among solid organ recipients ${ }^{21-23}$ and $31 \%$ in intestinal and multivisceral organ transplant recipients ${ }^{24}$. GCV resistance is $7.9 \%$ in HCT recipients from matched related or unrelated donors ${ }^{22,25}$ and $14.5 \%$ in high-risk patients ${ }^{26}$. GCV resistance in HIV-infected patients is reported to be $19.5 \% 27$.

Common mechanisms of CMV resistance to ganciclovir have been described chiefly with UL97 mutations. GCV is an acyclic nucleoside analogue of 2'-deoxyguanosine. In a multistep process dependent on both viral and cellular enzymes, GCV is converted to ganciclovir triphosphate, the chemical form that is active against CMV. The initial phosphorylation is catalyzed by an unusual protein kinase homolog encoded by the CMV UL97 open reading frame. Resistance to GCV arises from mutations in the UL97 gene. In several reports, numerous GCV-related mutations have been described. Most UL97 mutations conferring GCV resistance are strongly clustered at codons 460,520 , or 590 to $607^{16,28,29}$. In daily medical care, timely results of resistance testing would be useful for making clinical decisions. If no drug resistance is identified, clinical management may focus on improving host defenses rather than switching antivirals. If there is confirmed genotypic evidence of resistance, the specific mutation, host immune status, and disease severity should all factor into these decisions, to continue or intensify current treatment, to switch to a non-cross-resistant drug, to use drug combinations, or to try experimental drugs. Management algorithms have been proposed by several groups ${ }^{30}$.

In this study, we did not detect the mutations of the UL97 gene associated with GCV resistance. Our results might indicate that the uniform use of GCV is appropriate for initial antiviral therapy in CMV colitis associated with or without UC in GCV-naive patients, when antiviral therapy is needed. Since the sample size in our study was small, further study with large sample size is necessary to comfirm it. On the other hand, several mutations of the UL97 gene not associated with GCV resistance were detected, such as Q126L (100\%), T75A (93.3\%), and D605E (80\%). The D605E variant of UL97 was first described in 1 of 8 CMV isolates from an immunocompromised host in France ${ }^{31}$, but it has not been commonly observed in the human CMV strains circulating in western countries. Some reports have shown that the D605E mutation has frequently been detected in only Asian countries, and is estimated at $91.8 \%$ in Japanese infants and children and $78 \%$ in Chinese transplant recipients ${ }^{17,18}$. Thus, they suggested that this mutation could be an important molecular marker of CMV evolution in East Asian countries. Q126L, T75A, and D605E mutations, which were detected in this study, could potentially be used as genetic markers for CMV in East Asian countries. 
There are several limitations in this preliminary study. First, this study included a small number of patients, all of whom were GCV-naive patients. Therefore, the results should be interpreted only in GCVnaive patients. It is speculated that a high rate of UL97 mutations may be identified in patients who have been treated with GCV for a longer period. Second, we used the Sanger sequence to detect the mutations in this study. This cannot detect mutants that are present in $<10-20 \%$ of the viral population. Recently, several studies have been reported which detected the mutation of the CMV gene using a next-generation sequence (NGS) ${ }^{32,33}$. NGS methods have an improved ability to detect mixed populations and have been used to assess low-abundance variants. Third, we did not assess the mutation of DNA polymerase UL54 gene in this study, which is related to GCV resistance and cross-resistance to other antiviral drugs ${ }^{34}$. Therefore, further studies using NGS targeting both UL97 and UL54 in patients who have been treated with GCV are expected to provide more reliable evidence than this study.

Recognizing these limitations, the implications of our report are that no drug-resistant CMV strains were detected in the mucosa of the colon in patients with gastrointestinal CMV reactivation without a history of GCV administration, suggesting that it might not be necessary to consider CMV drug resistance at treatment initiation. In addition, these results were similarly observed when associated with UC and nonUC disease. Furthermore, the Q126L, T75A, and D605E mutations could potentially be used as genetic markers for CMV in East Asian countries. We look forward to further research in this area.

\section{Declarations}

\section{Compliance with Ethical Standards}

Conflict of interest: The authors declare that they have no conflict of interest.

Funding: This research was supported by Grants-in-Aid for Scientific Research (C) $19 \mathrm{~K} 08466$ from the Ministry of Education, Culture, Sports, Science and Technology, Japan.

Authors' contributions: ST and SO contributed to the study concept and design, analysis, and interpretation of data and drafting of the manuscript. ST performed all experiments for DNA sequencing. $\mathrm{NI}, \mathrm{TM}, \mathrm{ST}, \mathrm{YH}, \mathrm{MY}$, and MI contributed to patient management and acquisition of data and patient characteristics. IK was involved in histopathological diagnosis and advised on the methodology of the study. TF was involved in study supervision. KS and SO critically revised the manuscript for important intellectual content. All authors approved the final manuscript version prior to submission.

\section{References}


1. Ramanan, P. \& Razonable, R. R. Cytomegalovirus infections in solid organ transplantation: a review. Infect Chemother. 45, 260-271 (2013).

2. Razonable, R. R. Strategies for managing cytomegalovirus in transplant recipients. Expert Opin Pharmacother. 11, 1983-1997 (2010).

3. Mourad, F. H., Hashash, J. G., Kariyawasam, V. C. \& Leong, R. W. Ulcerative Colitis and Cytomegalovirus Infection: From A to Z.J Crohns Colitis(2020).

4. Rafailidis, P. I., Mourtzoukou, E. G., Varbobitis, I. C. \& Falagas, M. E. Severe cytomegalovirus infection in apparently immunocompetent patients: a systematic review. Virol J. 5, 47 (2008).

5. Yokoyama, Y. et al. Current Diagnostic and Therapeutic Approaches to Cytomegalovirus Infections in Ulcerative Colitis Patients Based on Clinical and Basic Research Data.Int J Mol Sci21(2020).

6. Roblin, X. et al. Cytomegalovirus load in inflamed intestinal tissue is predictive of resistance to immunosuppressive therapy in ulcerative colitis. Am J Gastroenterol. 106, 2001-2008 (2011).

7. Clos-Parals, A. et al. Prognostic Value of the Burden of Cytomegalovirus Colonic Reactivation Evaluated by Immunohistochemical Staining in Patients with Active Ulcerative Colitis. J Crohns Colitis. 13, 385-388 (2019).

8. Pillet, S. et al. Infliximab Does Not Worsen Outcomes During Flare-ups Associated with Cytomegalovirus Infection in Patients with Ulcerative Colitis. Inflamm Bowel Dis. 21, 1580-1586 (2015).

9. Pillet, S., Pozzetto, B. \& Roblin, X. Cytomegalovirus and ulcerative colitis: Place of antiviral therapy. World J Gastroenterol. 22, 2030-2045 (2016).

10. Lurain, N. S. \& Chou, S. Antiviral drug resistance of human cytomegalovirus. Clin Microbiol Rev. 23, 689-712 (2010).

11. Kotton, C. N. Updates on antiviral drugs for cytomegalovirus prevention and treatment. Curr Opin Organ Transplant. 24, 469-475 (2019).

12. Erice, A. et al. Antiviral susceptibilities and analysis of UL 97 and DNA polymerase sequences of clinical cytomegalovirus isolates from immunocompromised patients. J Infect Dis. 175, 1087-1092 (1997).

13. Gilbert, C. \& Boivin, G. Human cytomegalovirus resistance to antiviral drugs. Antimicrob Agents Chemother. 49, 873-883 (2005).

14. Chou, S. Antiviral drug resistance in human cytomegalovirus. Transpl Infect Dis. 1, 105-114 (1999).

15. Chou, S. et al. Cytomegalovirus UL97 phosphotransferase mutations that affect susceptibility to ganciclovir. J Infect Dis. 185, 162-169 (2002).

16. Chou, S. Advances in the genotypic diagnosis of cytomegalovirus antiviral drug resistance. Antiviral Res. 176, 104711 (2020).

17. Tanaka, K. et al. Human cytomegalovirus UL97 D605E polymorphism has a high prevalence in immunocompetent Japanese infants and children. Microbiol Immunol. 55, 328-330 (2011). 
18. Zhou, L., Fan, J., Zheng, S. S. \& Ma, W. H. Prevalence of human cytomegalovirus UL97 D605E mutation in transplant recipients in China. Transplant Proc. 38, 2926-2928 (2006).

19. Kotton, C. N. et al. The Third International Consensus Guidelines on the Management of Cytomegalovirus in Solid-organ Transplantation. Transplantation. 102, 900-931 (2018).

20. Ljungman, P. et al. Definitions of Cytomegalovirus Infection and Disease in Transplant Patients for Use in Clinical Trials. Clin Infect Dis. 64, 87-91 (2017).

21. Boivin, G. et al. Cytomegalovirus resistance in solid organ transplant recipients treated with intravenous ganciclovir or oral valganciclovir. Antivir Ther. 14, 697-704 (2009).

22. Hantz, S. et al. Drug-resistant cytomegalovirus in transplant recipients: a French cohort study. $J$ Antimicrob Chemother. 65, 2628-2640 (2010).

23. Young, P. G. et al. Ganciclovir-resistant cytomegalovirus infection in solid organ transplant recipients: a single-center retrospective cohort study. Transpl Infect Dis. 18, 390-395 (2016).

24. Ambrose, T. et al. Cytomegalovirus Infection and Rates of Antiviral Resistance Following Intestinal and Multivisceral Transplantation. Transplant Proc. 48, 492-496 (2016).

25. Allice, T. et al. Valganciclovir as pre-emptive therapy for cytomegalovirus infection post-allogenic stem cell transplantation: implications for the emergence of drug-resistant cytomegalovirus. $J$ Antimicrob Chemother. 63, 600-608 (2009).

26. Shmueli, E. et al. High rate of cytomegalovirus drug resistance among patients receiving preemptive antiviral treatment after haploidentical stem cell transplantation. J Infect Dis. 209, 557-561 (2014).

27. Azimi, T., Tavakolian, S., Goudarzi, H., Pourmand, M. R. \& Faghihloo, E. Global estimate of phenotypic and genotypic ganciclovir resistance in cytomegalovirus infections among HIV and organ transplant patients; A systematic review and meta-analysis. Microb Pathog. 141, 104012 (2020).

28. Campos, A. B., Ribeiro, J., Boutolleau, D. \& Sousa, H. Human cytomegalovirus antiviral drug resistance in hematopoietic stem cell transplantation: current state of the art. Rev Med Virol. 26, 161-182 (2016).

29. Chou, S., Guentzel, S., Michels, K. R., Miner, R. C. \& Drew, W. L. Frequency of UL 97 phosphotransferase mutations related to ganciclovir resistance in clinical cytomegalovirus isolates. $J$ Infect Dis. 172, 239-242 (1995).

30. El Chaer, F., Shah, D. P. \& Chemaly, R. F. How I treat resistant cytomegalovirus infection in hematopoietic cell transplantation recipients. Blood. 128, 2624-2636 (2016).

31. Alain, S. et al. Value of a new rapid non-radioactive sequencing method for analysis of the cytomegalovirus UL97 gene in ganciclovir-resistant strains. J Virol Methods. 51, 241-251 (1995).

32. Lopez-Aladid, R. et al. Improvement in detecting cytomegalovirus drug resistance mutations in solid organ transplant recipients with suspected resistance using next generation sequencing. PLoS One. 14, e0219701 (2019).

33. Sahoo, M. K. et al. Detection of cytomegalovirus drug resistance mutations by next-generation sequencing. J Clin Microbiol. 51, 3700-3710 (2013). 
34. Gilbert, C., Azzi, A., Goyette, N., Lin, S. X. \& Boivin, G. Recombinant phenotyping of cytomegalovirus UL54 mutations that emerged during cell passages in the presence of either ganciclovir or foscarnet. Antimicrob Agents Chemother. 55, 4019-4027 (2011).

\section{Tables}

Table 1. Table 1 Primer sets used in the nested PCR for this study

\begin{tabular}{lllcc}
\hline Primer name & \multicolumn{2}{c}{ Sequence (5'-3') } & Nucleotides Product length (bp) \\
\hline 1st round PCR & Forward & Reverse & \\
UL97 N1 & GTGCAGCCCTAGGAACA & CGTTTCTTCGAGCACC & $-35---637$ & 708 \\
UL97 N2 & GGTTACCACGGCTTGC & GCGGTAGCTCTCGTCG & $433---1008$ & 575 \\
UL97 N3 & GGTCTACGTGCCCAAAGA & GAAAGACGGCCACACAG & $933---1498$ & 565 \\
UL97 N3 & GTGCGATTACAGCCTCAG & GCGTCCAGGTTACTCG & $1437---2133$ & 696 \\
\hline 2nd round PCR & & & \\
UL97_1 & CTGTCGCCACTATGTCCTCC & GAACGCATGCGGAAAAAGTC & $-11---300$ & 311 \\
UL97_2 & CCACTTTGACCACCCTGAGT & GTGAAGAGAGCGCGGCGTA & $248---512$ & 264 \\
UL97_3 & CGTTCGAGTACGATCGCGAC GTCGTTGGAACAGGTGCAAT & $476---812$ & 336 \\
UL97_4 & CGGAAAGTCAGGACAGCG & CTCGTCGCTCATGTCCAC & $689---982$ & 293 \\
UL97_5 & GGACGATTTTGCCACAAGA CCACTGGTCGTGATGAAACA & $951---1253$ & 302 \\
UL97_6 & CTGCTGCACAACGTCACGGT CACAGCGCTCGTTGTAATCC & $1210---1467$ & 257 \\
UL97_7 & GATTACAGCCTCAGCGAGCC CATGCGCACCTCGTCCAG & $1441---1717$ & 276 \\
UL97_8 & GTAACGTGCTGGGCTTTTCC GCATTCGTGGTAGAAGCGGC & $1670---1949$ & 279 \\
UL97_9 & CCAAGATGTCCTCGTGTCGC CCAGGTTACTCGGGGAACAG & $1916---2129$ & 184 \\
\hline
\end{tabular}

Table 2. Enrolled patients and their primary diseases

\begin{tabular}{ll}
\hline Number of patients, $\mathrm{n}$ & 22 \\
Sex, men/women & $13 / 9$ \\
Age, $\mathrm{y}$, mean \pm SD (range) & $59.5 \pm 16.0(17-81)$ \\
\hline Primary diseases, $\mathrm{n}(\%)$ & \\
Ulcerative colitis & $15(68.2)$ \\
Non-ulcerative colitis & $7(31.8)$ \\
Malignant melanoma & $1(4.5)$ \\
Myelodysplastic syndrome & $1(4.5)$ \\
Sarcoidosis & $1(4.5)$ \\
Ischemic colitis & $1(4.5)$ \\
Autoimmune hepatitis & $1(4.5)$ \\
Hemodialysis & $1(4.5)$ \\
No disease & $1(4.5)$ \\
\hline
\end{tabular}

Table 3. Comparison of the patient characteristics between the UC and non-UC groups 


\begin{tabular}{lllll}
\hline & Overall & UC & Non-UC & Statistical analysis \\
\hline Number of patients, $\mathrm{n}$ & 22 & 15 & 7 & \\
\hline Sex, men/women & $13 / 9$ & $9 / 6$ & $4 / 3$ & $\mathrm{NS}$ \\
\hline Age; mean \pm SD (range), years & $59.5 \pm 16.0$ & $56.1 \pm 17.8$ & $66.9 \pm 8.4$ & $\mathrm{NS}$ \\
& $017-81 \square$ & $(17-81)$ & $(53-76)$ & \\
\hline Height, cm, mean \pm SD & $161.0 \pm 8.7$ & $160.9 \pm 8.3$ & $161.4 \pm 10.0$ & $\mathrm{NS}$ \\
\hline Weight, kg, mean \pm SD & $52.8 \pm 8.6$ & $52.7 \pm 9.9$ & $52.9 \pm 5.5$ & $\mathrm{NS}$ \\
\hline Body mass index, mean \pm SD & $20.4 \pm 3.1$ & $20.3 \pm 3.1$ & $20.5 \pm 3.5$ & $\mathrm{NS}$ \\
\hline Serum albumin, g/dl, mean \pm SD & $3.11 \pm 0.77$ & $3.42 \pm 0.65$ & $2.46 \pm 0.59$ & $P<0.01$ \\
\hline Serum CRP, mg/dl, mean \pm SD & $2.43 \pm 2.21$ & $2.15 \pm 2.45$ & $3.01 \pm 1.59$ & $\mathrm{NS}$ \\
\hline Hemoglobin, g/dl, mean \pm SD & $11.9 \pm 2.4$ & $12.3 \pm 2.6$ & $11.0 \pm 1.7$ & $\mathrm{NS}$ \\
\hline Total cholesterol, mg/dl, mean \pm SD & $163 \pm 45$ & $157 \pm 36$ & $171 \pm 56$ & $\mathrm{NS}$ \\
\hline Triglyceride, mg/dl, mean $\pm \mathrm{SD}$ & $121 \pm 50$ & $106 \pm 39$ & $140 \pm 60$ & $\mathrm{NS}$ \\
\hline Treatment, $\mathrm{n}$ (\%) & & & & \\
Oral or intravenous steroids & $8(36.4)$ & $5(33.3)$ & $3(42.9)$ & $\mathrm{NS}$ \\
Immunosuppressive agents & $9(40.9)$ & $7(46.7)$ & $2(28.6)$ & $\mathrm{NS}$ \\
Anti-TNF agents & $5(22.7)$ & $5(33.3)$ & $0(0)$ & $\mathrm{NS}$ \\
5-ASA & $10(45.5)$ & $10(66.7)$ & $0(0)$ & $P<0.01$ \\
Immune checkpoint inhibitor & $1(4.5)$ & $0(0)$ & $1(14.3)$ & $\mathrm{NS}$ \\
Other & $8(36.4)$ & $5(33.3)$ & $3(42.9)$ & $\mathrm{NS}$ \\
\hline Diagnosis of CMV infection, $\mathrm{n}(\%)$ & & & & \\
Histological H\&E staining & $4(18.2)$ & $0(0)$ & $4(57.1)$ & $P<0.01$ \\
Histological IHC staining & $19(86.4)$ & $13(86.7)$ & $6(85.7)$ & $\mathrm{NS}$ \\
Tissue CMV DNA PCR & $22(100)$ & $15(100)$ & $7(100)$ & $\mathrm{NS}$ \\
\hline
\end{tabular}

H\&E; hematoxylin and eosin, IHC; immunohistochemistry

Table 4 UL97 mutations in 22 Japanese patients with CMV colitis

\begin{tabular}{|c|c|c|c|c|c|c|c|}
\hline \multirow{2}{*}{$\begin{array}{c}\text { Amino acid } \\
\text { changes }\end{array}$} & \multicolumn{2}{|c|}{ Overall } & \multicolumn{2}{|c|}{$\mathrm{UC}$} & \multicolumn{2}{|c|}{ Non-UC } & \multirow{2}{*}{$\begin{array}{c}\text { Statistical } \\
\text { analysis }\end{array}$} \\
\hline & $\begin{array}{l}\text { No. of } \\
\text { strains }\end{array}$ & $\begin{array}{c}\% \text { of } \\
\text { strains }\end{array}$ & $\begin{array}{l}\text { No. of } \\
\text { strains }\end{array}$ & $\begin{array}{c}\% \text { of } \\
\text { strains }\end{array}$ & $\begin{array}{l}\text { No. of } \\
\text { strains }\end{array}$ & $\begin{array}{c}\% \text { of } \\
\text { strains }\end{array}$ & \\
\hline A53S & 1 & 4.5 & 1 & 6.7 & 0 & 0 & NS \\
\hline $\mathrm{T} 75 \mathrm{~A}$ & 21 & 95.5 & 14 & 93.3 & 7 & 100 & NS \\
\hline Q126L & 19 & 86.4 & 12 & 80.0 & 7 & 100 & NS \\
\hline R137C & 1 & 4.5 & 0 & 0 & 1 & 14.3 & NS \\
\hline $\mathrm{A} 140 \mathrm{~V}$ & 1 & 4.5 & 1 & 6.7 & 0 & 0 & NS \\
\hline G188S & 1 & 4.5 & 0 & 0 & 1 & 14.3 & NS \\
\hline L228P & 2 & 9.0 & 1 & 6.7 & 1 & 14.3 & NS \\
\hline D263G & 2 & 9.0 & 1 & 6.7 & 1 & 14.3 & NS \\
\hline D605E & 19 & 86.4 & 14 & 93.3 & 5 & 71.4 & NS \\
\hline $\mathrm{A} 674 \mathrm{~T}$ & 1 & 4.5 & 0 & 0 & 1 & 14.3 & NS \\
\hline T675A & 1 & 4.5 & 1 & 6.7 & 0 & 0 & NS \\
\hline
\end{tabular}

\section{List Of Abbreviations}


CMV, Cytomegalovirus; UC, ulcerative colitis; GCV, Ganciclovir; HIV, human immunodeficiency virus; PCR, polymerase chain reaction; DNA, deoxyribonucleic acid; NGS, next-generation sequencing.

\section{Figures}

Second PCR

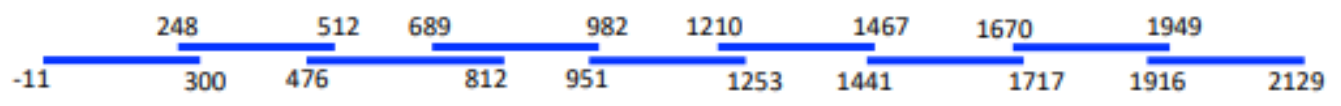

First PCR

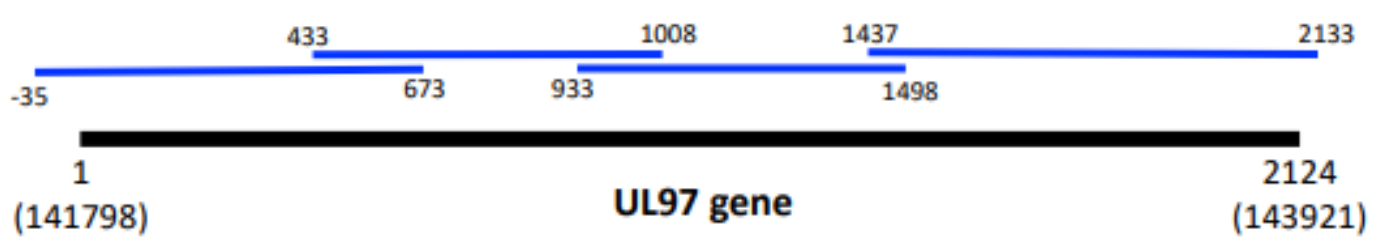

Figure 1

Diagram of nested PCR primer sites for UL97 gene analysis to determine GCV resistance mutation. The full length of UL97 gene (2123bp) is presented at the bottom. The initial nested PCR primer sites are represented in the lower row. The second PCR primer sites for DNA sequencing are represented in the upper row.

a

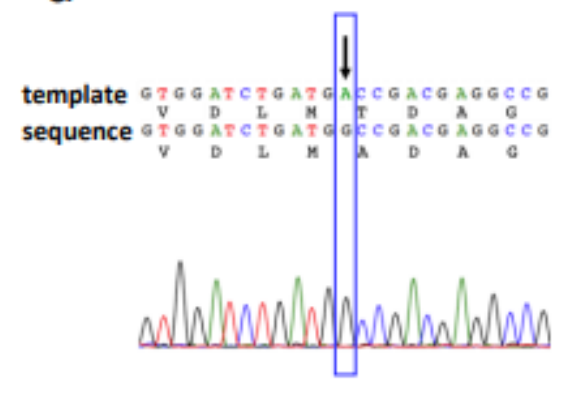

b

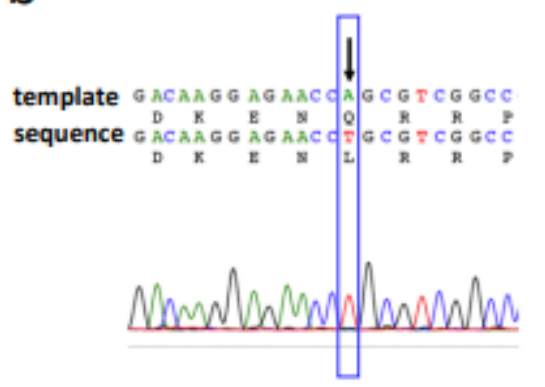

C

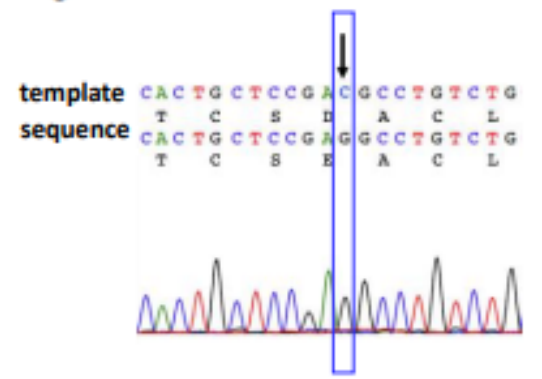

Figure 2

Typical results of the identified UL97 genetic mutations. (a) T75A, (b) Q126L, and (c) D605E

\section{Supplementary Files}

This is a list of supplementary files associated with this preprint. Click to download.

- TamuraSetal.SupplementaryTableS1ScientificReports.pdf 